\title{
TerraSAR-X Staring Spotlight Mode Optimization and Global Performance Predictions
}

\author{
Thomas Kraus, Benjamin Bräutigam, Josef Mittermayer, Steffen Wollstadt, and Christo Grigorov
}

\begin{abstract}
For the TerraSAR-X mission, a new staring spotlight mode has been implemented delivering very high azimuth resolution. Detailed performance analyses have been conducted to optimize the commanding parameters for this mode. Compared to the previously available TerraSAR-X imaging modes, staring spotlight requires operating the instrument with minimum margins and at the edge of the specifications, the radar has been designed for. Therefore, an additional step-a global acquisition simulation and analysis-is introduced during the operationalization to ensure the suitability of the derived commanding parameters on a global scale. This paper gives an overview of SAR performance analyses conducted for the mode optimization and implementation phases and presents a novel global performance assessment approach, which is generally applicable for the verification of operational SAR modes. Additionally, measurement results and exemplary acquisitions are shown.
\end{abstract}

Index Terms-High resolution, staring spotlight, synthetic aperture radar (SAR), TerraSAR-X.

\section{INTRODUCTION}

D URING 2013, the portfolio of the TerraSAR-X mission radar-imaging modes has been substantially extended. Since the launch of TerraSAR-X in 2007, four SAR imaging modes have been operationally available (cf. Table I). They range from sliding spotlight modes called spotlight (SL) and high-resolution spotlight (HS) with azimuth resolutions down to $1.1 \mathrm{~m}$, over stripmap (SM), to a four-beam scanSAR (SC) mode with a range coverage of $100 \mathrm{~km}$. In order to make new applications accessible, two new modes have been developed and are operationally available since autumn 2013 [1], [2]. These are a wide ScanSAR mode with a range coverage of more than $200 \mathrm{~km}$ for ship detection and sea ice monitoring, and a staring spotlight (ST) mode with an azimuth resolution of $24 \mathrm{~cm}$ for applications like airfield surveillance and critical infrastructure monitoring. This mode allows an identification of even small-scale objects and an easier visual interpretation of the SAR imagery.

Spotlight modes generally deliver an increased azimuth resolution compared to stripmap acquisitions. The beam is steered backward relative to the direction of flight to increase the aperture time and thus the azimuth signal bandwidth of each

Manuscript received October 31, 2014; revised March 20, 2015; accepted April 27, 2015. Date of publication June 10, 2015; date of current version February 22, 2016. TerraSAR-X is supported by the German Federal Ministry for Economic Affairs and Energy (50 EE 1328) and realized in a public-private partnership between DLR and AIRBUS Defence \& Space.

The authors are with the Microwaves and Radar Institute, German Aerospace Center (DLR), 82234 Oberpfaffenhofen, Germany (e-mail: T.Kraus@dlr.de).

Color versions of one or more of the figures in this paper are available online at http://ieeexplore.ieee.org.

Digital Object Identifier 10.1109/JSTARS.2015.2431821 target within the scene. The increased bandwidth improves the azimuth resolution compared to a stripmap acquisition. However, in sliding spotlight modes (e.g., SL and HS), the main lobe of the azimuth antenna pattern is still sweeping forward over the scene in the azimuth direction, even though slower as in stripmap. This is shown on the left of Fig. 1. In the staring spotlight mode, the goal of the antenna steering is to maintain the scene center in the center of the main lobe of the azimuth beam for the whole aperture time, as depicted on the right of Fig. 1. In contrast to the sliding spotlight modes, the antenna beam in staring spotlight is directly pointing to the center of the scene during the entire data acquisition. This maximizes the target azimuth bandwidth for a given acquisition duration and leads to the maximum possible azimuth resolution at the cost of a smaller scene extent in azimuth [3].

For the TerraSAR-X staring spotlight mode, the azimuth antenna steering angle is significantly larger than for the sliding spotlight modes, enabling higher resolution images but causing a considerably increased range cell migration (RCM). TerraSAR-X employs an active phased array antenna with an azimuth element spacing greater than the wavelength. Therefore, the performance of electronically steered (off-boresight) azimuth beams is affected by grating lobes. The effect is increasing for greater steering angles [4], as used for staring spotlight. Thus, the azimuth ambiguity performance has been identified to be the driving performance parameter for staring spotlight [5], [6]. An optimized selection of the pulse repetition frequency (PRF) is therefore essential.

Besides the increased antenna steering span compared to the sliding spotlight modes, the maximum possible range chirp bandwidth of $300 \mathrm{MHz}$ is employed to maximize the range resolution as far as possible for TerraSAR-X to be closer to the high azimuth resolution. However, the high bandwidth causes a high data rate that is limiting the receive echo window length, and therefore the range scene size due to the instrument constraints buffer length and data rate limitations [1], [5], [7]. The specified range extent of the mode-called nominal swath width-is further limited since margins are necessary to cope with orbit variations and inaccuracies in the digital elevation model (DEM) used for the command generation as well as for losses due to the RCM correction.

The staring spotlight mode of TerraSAR-X is required to be operationally available for worldwide acquisition. Therefore, the commanding parameters and especially the PRF has to be optimized and their suitability has to be validated on a global scale. The optimized staring spotlight products shall complement and shall be well differentiated from the other well-established and recognized TerraSAR-X products. Due 
TABLE I

OpERATIONAL TERRASAR-X IMAGING MODES

\begin{tabular}{|l|r|r|r|}
\hline Mode & $\begin{array}{r}\text { Range } \\
\text { scene size }(\mathrm{km})\end{array}$ & $\begin{array}{r}\text { Azimuth } \\
\text { resolution }(\mathrm{m})\end{array}$ & $\begin{array}{r}\text { Azimuth } \\
\text { size }(\mathrm{km})\end{array}$ \\
\hline scene & $\approx 5$ & 0.24 & $\approx 2.5$ \\
\hline Staring spotlight & 10 & $1.7 / 1.1$ & $10 / 5$ \\
\hline Spotlight (SL/HS) & 30 & 3.3 & 50 \\
\hline Stripmap & 100 & 18.5 & 150 \\
\hline ScanSAR (four beams) & $\geq 200$ & 40.0 & 200 \\
\hline ScanSAR (six beams) & & &
\end{tabular}

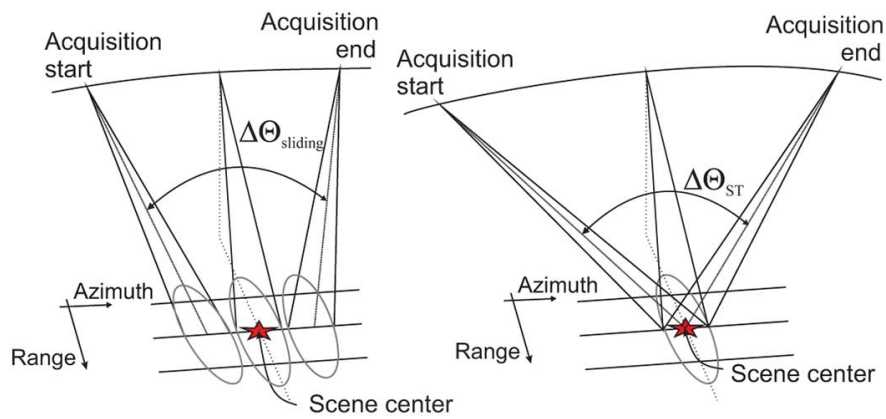

Fig. 1. Acquisition geometry of a SAR operating in a sliding spotlight mode, e.g., high-resolution spotlight (left) and in staring spotlight mode (right).

to the azimuth antenna steering beyond the design limits, the range of PRFs delivering high-performance products drastically shrinks. However, in order to cope with satellite altitude and topographic variations, the echo window timing and the PRF have to be adapted for each individual acquisition scenario. Hence, a second step after the selection of PRFs is necessarythe proof of the suitability for commanding and the compliance with performance requirements for acquisitions all over the Earth.

This paper focuses on the optimization of the TerraSAR-X staring spotlight mode and a performance prediction approach based on the simulation of globally distributed acquisitions. From the optimization step, the global simulations and the evaluation of really acquired staring spotlight images, mode specifications are derived. Although the operational TerraSAR-X commanding algorithms are incorporated in the simulation environment, they are not addressed in detail here. More information for this can be found in [8].

The outline of this paper is as follows. In Section II, the key challenges of the staring spotlight mode of TerraSAR-X are addressed and the strategy for the optimization of commanding parameters is developed. Section III introduces a novel performance assessment approach based on global-scale acquisition simulations. The expected ambiguity performance as well as other key parameters are derived from these simulations. TerraSAR-X image evaluation of impulse response function (IRF) parameters, the noise characteristics, and the radiometric resolution are shown in Section IV. In Section V, two of the very first operational staring spotlight acquisitions are compared to standard TerraSAR-X high-resolution spotlight images and important differences are addressed. A summary of the optimized key performance parameters of the operational staring spotlight mode is given in Section VI.

\section{Mode Optimization}

The starting point for the analysis described in this paper is a study on the capability to acquire TerraSAR-X SAR images with high azimuth resolution in staring spotlight mode [5], [6], [9], [10]. For achieving a drastic improvement in azimuth resolution, the recommendation of the study is to increase the azimuth antenna steering span $\Delta \Theta_{\mathrm{ST}}$ (cf. Fig. 1 on the right) of the acquisition to $4.42^{\circ}$.

In [5], the azimuth antenna characteristics of TerraSAR-X are treated in detail. It is shown that the high grating lobe gain, especially of steered azimuth beams [4], leads to strong signal ambiguity contributions degrading the azimuth ambiguity-tosignal ratio (AASR) of the acquisition [11]. There are several options to improve the AASR. The theoretical possibility to reduce azimuth ambiguities by the use of an azimuth processing window and a proper PRF selection was demonstrated in [5]. For all TerraSAR-X modes, an azimuth processing window is used for sidelobe suppression [1], [12]. The same (generalized) Hamming window with $\alpha=0.6$ is used for staring spotlight. Additionally, it was shown that a reduction in the azimuth scene size also leads to an improvement of the overall AASR. As the distance to scene center increases, the AASR is degrading. If the outer targets are excluded from the image, the average as well as the worst-case AASR performance can be improved to a tolerable level. The careful selection of the PRF and the definition of the azimuth scene extent are the two options chosen for the optimization of the AASR performance of the TerraSAR-X staring spotlight mode.

The goal during the operational staring spotlight mode implementation was to derive optimized commanding parameters and to specify the resulting mode characteristics. There are 122 different elevation beams available on-board for staring spotlight, covering the incidence angle range from $15^{\circ}$ to $60^{\circ}$. For each elevation beam, different SAR parameters are necessary to achieve image products with optimized performance. The usable PRF range, the azimuth scene size, and margins for the range swath width have to be defined individually.

\section{A. Operational TerraSAR-X Commanding System}

During the operational mode implementation, a comprehensive performance simulation evolved from [5] has been conducted, dedicated to the actual, operational system. In order to make the simulations as accurate and realistic as possible, all inputs are taken from the operational TerraSAR-X ground segment environment, e.g.,

1) the reference orbit [13];

2) the attitude steering [14];

3) azimuth and elevation antenna patterns [15];

4) the on-board PRF configurations;

5) the quantized steering angles of the azimuth beams;

6) the finite number (125) of different azimuth beams which can be used during one acquisition;

7) a commanding sequence calculated by the operational commanding algorithms [8].

The operational commanding algorithms calculate radar instrument parameters like the PRF, the echo window timing, 


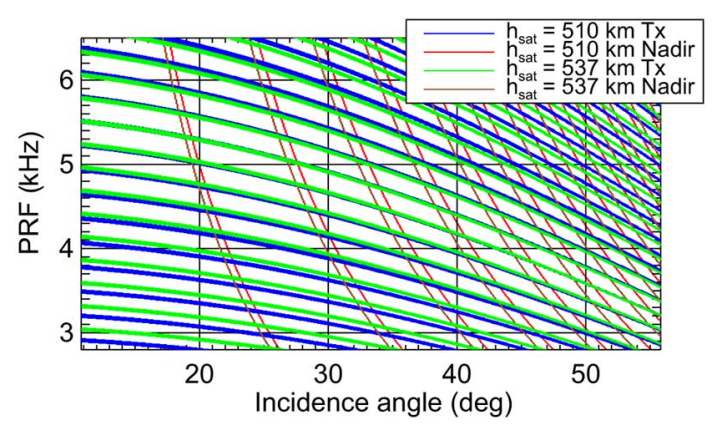

Fig. 2. TerraSAR-X timing diagram with nadir events and transmit events for a satellite orbit altitude of 510 and $537 \mathrm{~km}$, respectively. The white regions indicate suitable PRFs over certain incidence angle ranges.

receiver gain settings, and for spotlight modes, the azimuth antenna steering sequence individually for each scene on the ground to be imaged. The commanding software is integrated into the mission operations segment of the TerraSAR-X mission and running automatically for each requested acquisition [8]. The operational, autonomous character of this software causes high demands regarding reliability and computational efficiency that need to be fulfilled by the extension of the instrument commanding system to staring spotlight. In Section III, a novel approach is presented, utilizing the highly efficient operational algorithms in a simulation framework to predict the SAR performance on a global scale [2]. At the same time, the staring spotlight updated operational algorithms are verified to ensure a high reliability.

\section{B. PRF Optimization}

The driving factors for the selection of an adequate PRF for a SAR acquisition are the echo timing constraints and the ambiguity-to-signal ratio (ASR). The timing is limited by the transmit event and the return of nadir echoes. It is not possible to receive radar echoes during the transmission of a pulse, and the reception of nadir returns is not desirable. The power of the nadir return can be strong compared to the power of the return from the actually imaged scene. Fig. 2 depicts the nadir returns and transmit events as function of the incidence angle for the minimum and maximum satellite orbit altitudes of TerraSAR-X. White regions indicate incidence angle ranges that can be used for acquisition with dedicated PRFs. It can be seen from Fig. 2 that the choice of the PRF depends on the satellite altitude. The elevation of the target and nadir areas need to be considered as well. The usable incidence angle range with the corresponding PRF also determines the maximum possible swath width. Vice versa, a necessary swath width further limits the ranges of usable PRFs for a given incidence angle.

The strategy of the TerraSAR-X command generation system is to use a PRF as close as possible to a so-called target PRF. This target PRF is a compromise regarding the overall ASR performance, delivering comparable values for ambiguities in azimuth (AASR) and range (RASR) direction and thus minimizing the ASR. Neither the AASR nor the RASR should be dominating. Fig. 3 shows an example. The AASR and RASR curves for elevation beam spot_063 versus the PRF are depicted

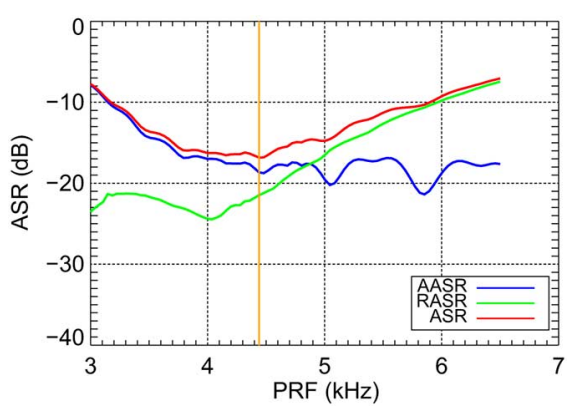

Fig. 3. Example for AASR and RASR trends versus PRF in blue and green, respectively. The AASR values represent a point target at an outer azimuth position of a staring spotlight scene. The RASR values are worst-case over all simulated range targets for a scene imaged with elevation beam spot_063 at an incidence angle of $44^{\circ}$. The red line represents the ASR value. Its minimum is marked by the orange vertical line, being the target PRF of approximately $4450 \mathrm{~Hz}$.

in blue and green, respectively. The red curve represents the ASR, i.e., the linear sum of the powers of both ambiguities. Its minimum is highlighted by the orange vertical line being the target PRF of approximately $4450 \mathrm{~Hz}$. The target PRF is enclosed by a minimum and a maximum PRF that still deliver acceptable AASR and RASR values. This PRF range provides a certain flexibility that is required to find timing parameters in the context of varying orbit altitude and scene topography [16].

A Ulaby X-band backscatter model for soil and rock [17] is used during the ambiguity calculations and the coupling between range and azimuth ambiguities is considered as negligible [18]. Fig. 4 shows the AASR for point targets uniformly distributed in azimuth direction of a staring spotlight scene versus PRF. For the simulation, a (generalized) Hamming window with $\alpha=0.6$ is used. Due to the strong grating lobes, an oscillating behavior of the AASR over the PRF is observable. The local minima occur for PRFs for which-integrated over the whole synthetic aperture and all azimuth beams - the ambiguities fall into low-gain regions of the respective azimuth antenna patterns. Additionally, the AASR performance degrades for targets with increasing distance to scene center as shown in Fig. 4. This AASR degradation occurs due to the fact that the azimuth antenna beam is continuously pointing with its highest gain toward the center of the scene. Targets with more azimuth offset to the scene center are observed with a lower gain part of the respective azimuth antenna pattern main lobe. Consequently, a reduction in the azimuth scene size can be used as a measure to avoid poor AASR performance at the azimuth scene edges.

Staring spotlight is a mode to acquire scenes with the best possible resolution, even at the cost of a relatively small scene size. The main area of interest-e.g., infrastructure that shall be monitored or objects to be detected and recognized-is assumed to be close to the center of the scene. Thus, the goal is to achieve the best possible ambiguity performance for areas close to scene center. A PRF close to a local minimum of the curves in Fig. 4 provides the best AASR at scene center and in approximation a symmetric degradation with increasing target azimuth offset from scene center. Thus, the local minima from Fig. 4 have to be selected as target PRF (indicated by red arrows). The possible target PRFs with their respective 


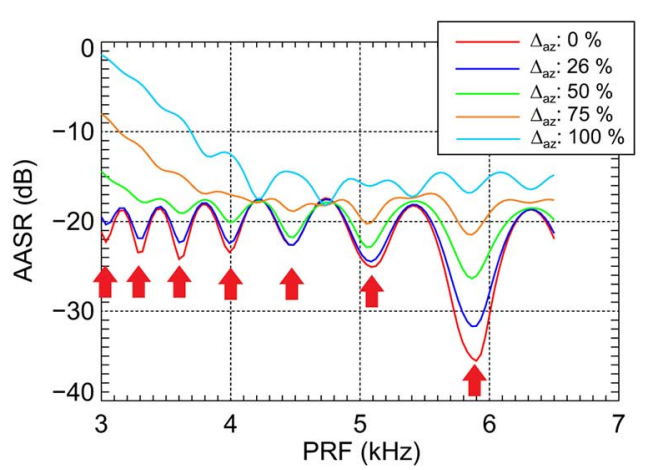

Fig. 4. Simulated AASR at different azimuth target positions. The legend represents the azimuth offset from scene center in percent of the projected 3-dB azimuth beam width on the ground. A Hamming window $(\alpha=0.6)$ was used. Red arrows indicate local minima of the AASR curves. Their corresponding PRFs are selected as target PRFs for the TerraSAR-X staring spotlight commanding. At the target PRFs, the AASR is increasing with increasing target offset from scene center.

TABLE II

Possible Target PRFs Derived From AASR Simulations For the TERRASAR-X STARING SPOTLIGHT COMMANDING AND THEIR Corresponding Minimum and MaXimum AASR Over the 3-DB ONE-WAY BEAM WIDTH

\begin{tabular}{|l|c|c|}
\hline PRF (Hz) & Minimum AASR (dB) & Maximum AASR (dB) \\
\hline 5860 & -35.4 & -16.2 \\
5100 & -25.0 & -15.8 \\
4450 & -22.6 & -14.4 \\
4000 & -23.3 & -12.4 \\
3600 & -23.9 & -8.2 \\
3300 & -23.5 & -4.5 \\
3050 & -21.8 & -1.8 \\
\hline
\end{tabular}

The AASR values are independent of the incidence angle.

minimum and maximum AASR values for a scene extent corresponding to the projection of the 3 -dB azimuth beam width on the ground are summarized in Table II. The selection of the local minimum for each individual elevation beam depends on the RASR performance of the beam. For steep incidence angle beams, a PRF of 5100 or $5860 \mathrm{~Hz}$ is possible since range ambiguities are not a concern. Due to further rising range ambiguities with increasing incidence angle, even the AASR minima of Fig. 4 at lower PRFs have to be used for elevation beams with an incidence angle of more than $45^{\circ}$. In Fig. 5, an optimized target PRF configuration based on ambiguity considerations is depicted. The target PRF stays at $5860 \mathrm{~Hz}$ up to an incidence angle of $36^{\circ}$. These PRFs do not represent the final configuration for the TerraSAR-X staring spotlight mode. The range swath width of TerraSAR-X products with $300 \mathrm{MHz}$ range chirp bandwidth is limited by instrument constraints, i.e., the echo buffer length and data rate limitations and therefore depends on the PRF. For this reason, the target PRF especially for medium incidence angles is lowered to achieve an adequate range scene size. Simulation results for the scene sizes are shown in Section III-B.

\section{Azimuth Scene Extent}

The staring spotlight mode always points to scene center. Thus, the azimuth scene extent corresponding to a certain

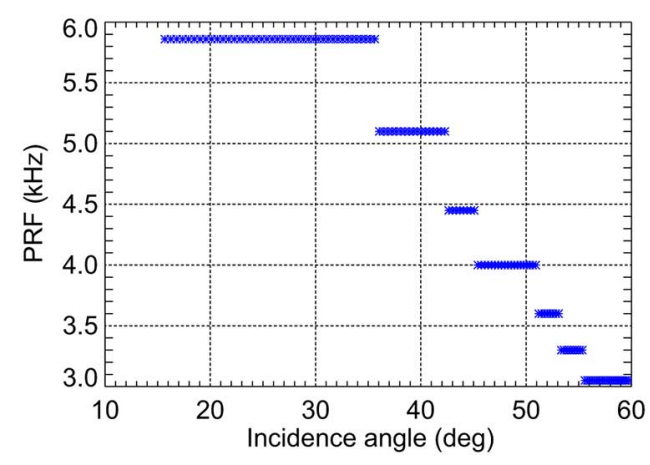

Fig. 5. Optimized target PRF configuration for the TerraSAR-X staring spotlight mode based on ambiguity considerations. The incidence angle range from $15^{\circ}$ to $60^{\circ}$ covers all 122 spotlight elevation beams.

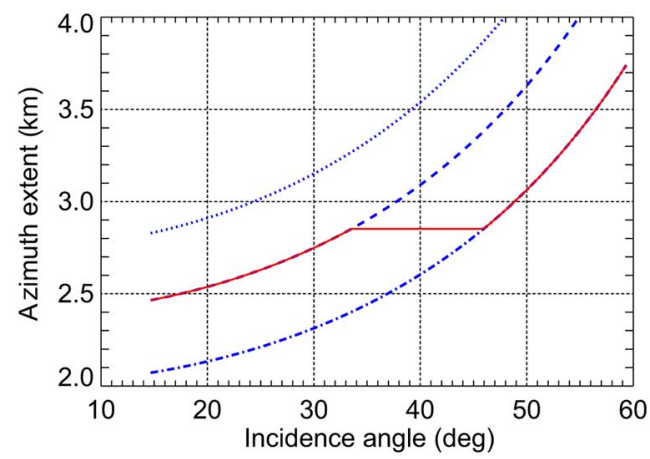

Fig. 6. Possible azimuth scene extent of staring spotlight acquisitions depending on the incidence angle. The dotted line represents the scene size which would be achieved if $100 \%$ of the ground-projected 3-dB (one-way) azimuth beam is used over the whole incidence angle range. The dashed and dasheddotted lines correspond to a percentage of $88 \%$ and $75 \%$, respectively. The red curve represents the finally specified azimuth scene extent for TerraSAR-X staring spotlight products.

fraction of the 3-dB azimuth beam width increases with larger incidence angle. The azimuth scene size of the TerraSAR-X staring spotlight products can be specified as a fraction of the area illuminated by the boresight azimuth beam. This beam is pointing perpendicular to the antenna aperture with respect to the azimuth direction. As mentioned earlier, 100\% correspond to the 3-dB one-way beam width. The actual values in kilometers as function of the incidence angle are depicted in Fig. 6 for $100 \%, 88 \%$, and $75 \%$ as dotted, dashed, and dashed-dotted blue lines, respectively.

Restrictions to the scene extent are introduced as a function of incidence angle to cope with rising azimuth ambiguities and decreasing NESZ performance for targets at outer azimuth positions. As discussed in Section II-B, the AASR performance and the achievable NESZ for steep incidence angles are better. Therefore, the selected percentage of the illuminated scene with sufficient SAR performance is larger in near range. This strategy also ensures a more homogeneous performance along the incidence angle. Following this approach, the azimuth scene size of staring spotlight products can be specified as indicated by the red curve in Fig. 6 . The scene size follows the $88 \%$ line up to an incidence angle of $34^{\circ}$. Up to this incidence angle, a target PRF of at least $5100 \mathrm{~Hz}$ is used, ensuring a good AASR performance even for target positions further away from scene 


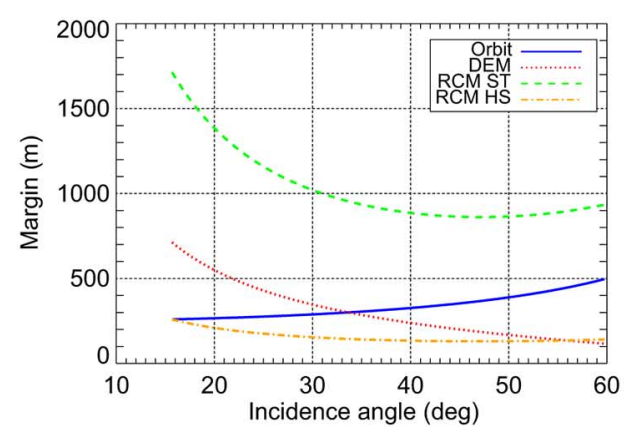

(a)

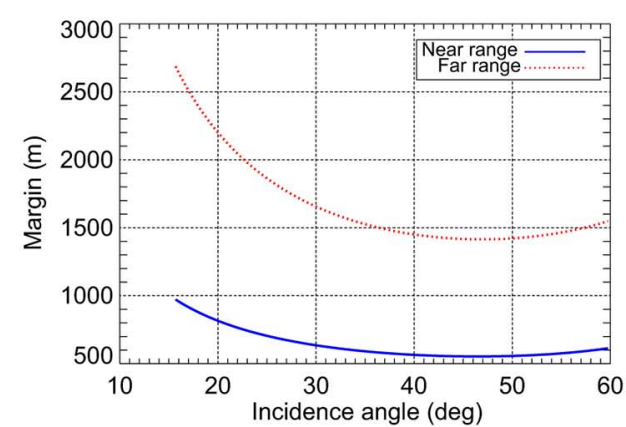

(b)

Fig. 7. (a) Swath width margin contributions (ground range) introduced during the calculation of the receive echo window to compensate orbit tube variations of $250 \mathrm{~m}$ (solid line), DEM inaccuracies of $200 \mathrm{~m}$ (dotted line), RCM for the steering angle span of an staring spotlight acquisition (dashed line), and for a high-resolution spotlight acquisition for comparison (dashed-dotted line). (b) Combined swath width margins (ground range) in near (solid line) and far range (dotted line) of staring spotlight acquisitions as a function of incidence angle.

center. For higher incidence angle beams, the azimuth scene size is kept constant at $2850 \mathrm{~m}$ until the $75 \%$ line is reached. For these beams, the target PRF is lowered to cope with rising range ambiguity levels (cf. Fig. 5). The resulting AASR degradation is partially compensated by a reduced fraction of the used beam width, keeping the width in kilometers constant. Finally, for larger incidence angles above $46^{\circ}$, the azimuth scene size follows the $75 \%$ line.

These optimizations together with the selected PRF ranges allow to achieve an ambiguity performance which is very homogeneous over a wide incidence angle range. The AASR and RASR of staring spotlight can thus be specified to be better than -17 and $-20 \mathrm{~dB}$, respectively, for the full performance range covering incidence angles up to $45^{\circ}$ [1].

\section{Margin Considerations}

There are several effects that can lead to a shift or degradation of the acquired scene in range, e.g., orbit variations and DEM errors [19]. The orbit of the satellite is subject to disturbances, e.g., due to atmospheric drag. Nevertheless, it is precisely controlled to be within a toroidal tube with a radius of $250 \mathrm{~m}(1-\sigma)$ relative to the reference orbit [20]. Each cross-track deviation of the actual orbit relative to the reference orbit — which is used for the timing calculations by the "radar command generation" algorithms [8] — leads to a shift of the acquired scene relative to the actually intended scene. Besides the satellite position, the topographic height of the scene is an essential input for the timing calculations. An error in the assumed topographic height also leads to a shift of the acquired scene relative to the intended one. The scene elevation information is retrieved from a DEM library containing data from the Shuttle Radar Topography Mission (SRTM) [21] and Global Land One-km Base Elevation (GLOBE) DEM data. The height error of the SRTM data is in the order of $10 \mathrm{~m}$ [22]. However, SRTM only covers areas up to $\pm 60^{\circ}$ latitude. For the rest of the world, GLOBE data have to be used that show errors of up to $300 \mathrm{~m}$ [23]. In order to cope with these orbit and DEM errors, margins are introduced during the timing calculations to be able to deliver the promised scene coverage. Introducing margins in this context means to enlarge the receiving echo window by an earlier opening and later closing. Fig. 7(a) depicts the ground range margins for an orbit variation (solid line) of $250 \mathrm{~m}$. This value corresponds to the requirement for the TerraSAR-X orbit control accuracy. By depicting the ground range margins in Fig. 7, the numbers can be compared with the ground range extent of the scenes (cf. Section III-B), highlighting the relatively large amount of margin necessary for the staring spotlight mode. An error margin of the DEM used for the timing calculations of $200 \mathrm{~m}$ is considered. This value is a compromise, ensuring the coverage of the intended scene with high probability, but at the same time keeping the margin values at a tolerable level. The same inputs have been used for the calculation of the margins for all other TerraSAR-X modes.

Besides these inaccuracies, the margins have to incorporate $\mathrm{RCM}[11]$

$$
\Delta R\left(\theta_{\mathrm{i}}\right)=R_{0}\left(\theta_{\mathrm{i}}\right) \cdot\left(\frac{1}{\sqrt{1-\left(\frac{f_{\mathrm{a} \text { max }} \cdot \lambda}{2 \cdot v_{\mathrm{eff}}}\right)^{2}}}-1\right) \cdot \frac{1}{\sin \left(\theta_{\mathrm{i}}\right)}
$$

where $\Delta R$ is RCM, $R_{0}$ is the range of closest approach, $f_{\mathrm{a}, \max }$ the maximum Doppler frequency of the acquisition, $v_{\text {eff }}$ the effective radar velocity, and $\theta_{\mathrm{i}}$ the incidence angle. Due to the larger azimuth steering span, the Doppler frequency for staring spotlight (ca. $\pm 19 \mathrm{kHz}$ ) is significantly larger than for the high-resolution spotlight mode. For a comparison, the RCM margins for both modes are depicted in Fig. 7(a) by the dashed and dashed-dotted lines. The prolongation of the receive echo window for the duration corresponding to the shown maximum ground range RCM is necessary since the echo window position is not adjusted during the whole aperture time of any spotlight acquisition of TerraSAR-X. To ensure a full resolution focusing for each point target within the scene, the echo window has to be extended in far-range direction since RCM leads to an increased range compared to the range of closest approach. Therefore, the total swath width margins in near and far range of a scene are asymmetric, which can be observed in Fig. 7(b). For the near range margin $m_{\text {near }}$, only orbit and DEM inaccuracies have to be taken into account by their respective margins $m_{\text {orbit }}$ and $m_{\text {DEM }}$

$$
m_{\text {near }}\left(\theta_{\mathrm{i}}\right)=m_{\text {orbit }}\left(\theta_{\mathrm{i}}\right)+m_{\mathrm{DEM}}\left(\theta_{\mathrm{i}}\right) .
$$




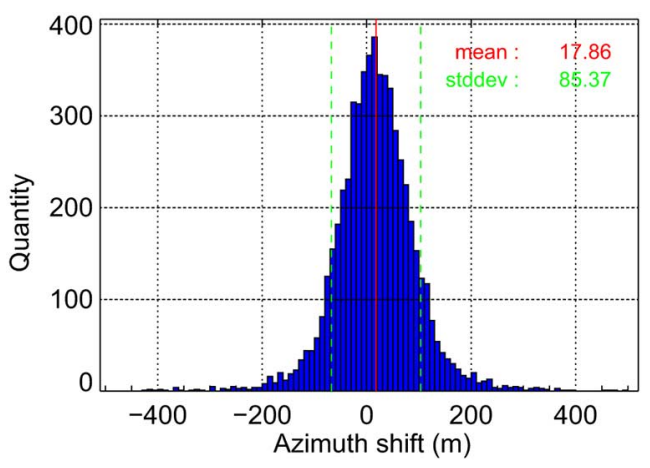

Fig. 8. Histogram of the measured along-track positioning error of more than 5000 real TerraSAR-X high-resolution spotlight acquisitions. The mean value is indicated as solid red vertical line and the dashed green vertical lines represent the mean value plus minus one standard deviation.

In far range, the margin to cope with RCM adds up and dominates the sum

$$
m_{\mathrm{far}}\left(\theta_{\mathrm{i}}\right)=m_{\mathrm{orbit}}\left(\theta_{\mathrm{i}}\right)+m_{\mathrm{DEM}}\left(\theta_{\mathrm{i}}\right)+\Delta R\left(\theta_{\mathrm{i}}\right)
$$

For the operational staring spotlight mode, the RCM is accounted for by the described margin as an extension to the echo window. Since the echo window length for acquisitions with a range chirp bandwidths of $300 \mathrm{MHz}$ is constrained by the instrument hardware architecture, this results in an effective shortening of the nominal swath width. However, it is possible to overcome this by shifting the echo window position with (azimuth) slow time, e.g., with each azimuth beam used. Since the introduced margin is larger than a kilometer in ground range (depending on the incidence angle), this method could improve the nominal swath width. The repositioning of the echo window with slow time is not implemented for the operational staring spotlight mode because it would drastically increase the command load. The instrument's state machine and the telecommand up-link to the spacecraft are the limiting factors for this approach.

TerraSAR-X is equipped with a GPS controlled on-board start time correction mechanism. This mechanism ensures a very accurate along-track positioning of acquisitions making a mode like staring spotlight with small azimuth scene sizes possible. However, the accuracy of this start time correction mechanism is also finite. This effect is accounted for by an azimuth scene size margin. The azimuth scene center shift of more than 5000 actual high-resolution spotlight acquisitions (with a range chirp bandwidth of $300 \mathrm{MHz}$ ) was analyzed by comparing the center of the acquired scene with the scene center coordinate used for the commanding. In Fig. 8, a histogram of the along-track error is shown. The histogram is symmetric with a mean value of about $18 \mathrm{~m}$ (solid red vertical line) and a standard deviation of approximately $85 \mathrm{~m}$ (dashed green vertical lines symmetric to the mean value). Therefore, a margin of $100 \mathrm{~m}$ in early and late azimuth is regarded adequate for staring spotlight. Due to this margin, the resulting azimuth scene sizes delivered to the user are $200 \mathrm{~m}$ larger than specified (cf. Fig. 6).

\section{Global Performance Prediction}

During the optimization step of the staring spotlight mode described in Section II, commanding parameters such as the azimuth scene size, scene size margins, and especially a PRF range have been derived for each individual elevation beam. In a further step, it is necessary to validate the derived commanding parameters on a global scale to estimate the impact of timing variations. The TerraSAR-X orbit altitude changes from 510 to $537 \mathrm{~km}$ above the WGS84 ellipsoid, and topographic variations further impact the timing conditions. Another important aspect from an operationalization point of view is the verification of the instrument command generation software [8] which had to be updated to support the staring spotlight commanding. Additionally, the goal was to assess key performance parameters based on realistic acquisition scenarios with data taken all over the Earth's land masses. All three aspects are covered within the framework of a novel global simulation and performance prediction approach.

\section{A. Global Simulation Approach}

In Fig. 9, the global performance simulation environment is depicted. It ranges from scene center coordinate selection over acquisition geometry and parameter calculation to the evaluation of the results. The middle block incorporates the operational algorithms for TerraSAR-X instrument commanding. The details of these algorithms are not addressed in this paper, but in [8]. However, their integration in the simulation environment is the main feature of the global performance assessment approach. This results in three main advantages. The simulation itself benefits from streamlined, computationally efficient algorithms enabling a global approach with a large set of simulated scenes. Furthermore, the operational commanding chain including the updates for the new staring spotlight mode is validated. To generate a huge amount of randomly placed acquisitions is an excellent test for the software and the used inputs. The third advantage which is stressed the most in this paper is the opportunity to generate performance estimates on a global scale that are as close as possible to the real-world system. Having the operational commanding software in the loop, no more simplifications are necessary and no detail is neglected concerning these parts of the overall SAR system.

In the first step of the simulation workflow, a scene center coordinate is randomly selected over the Earth's land masses. Fig. 10 shows the spatial distribution of more than 20000 scene center coordinates evaluated during the staring spotlight mode simulation. For each of these coordinates, all TerraSAR-X staring spotlight mode acquisition possibilities within the repeat cycle of 11 days are derived. Depending on the latitude of the coordinate, several options for an acquisition exist in ascending and descending geometry and with different incidence angles and therefore elevation beams. One of these possibilities is randomly selected. Fig. 11 shows a histogram of the elevation beams selected during the simulations. For each of the 122 elevation beams, more than 150 acquisitions have been simulated. For each scene, the operational TerraSAR-X instrument 


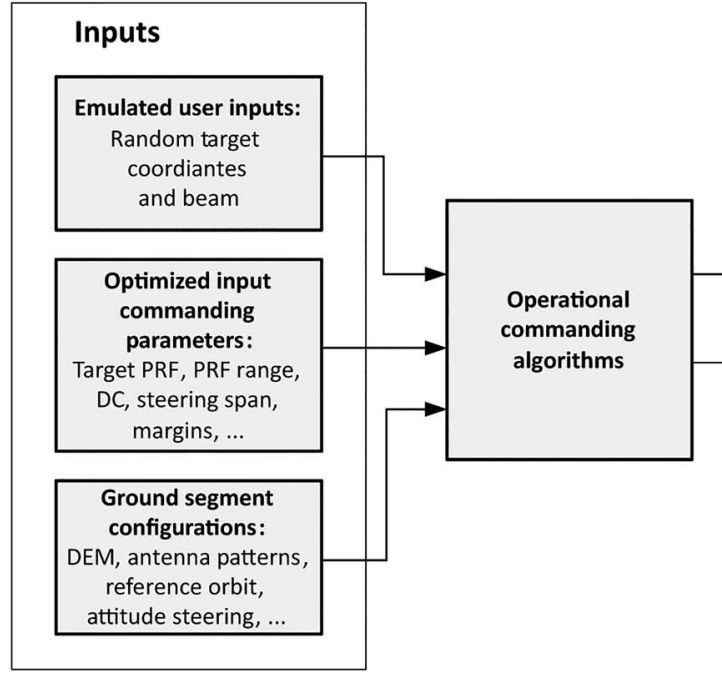

Fig. 9. Block diagram of the global performance simulation environment.

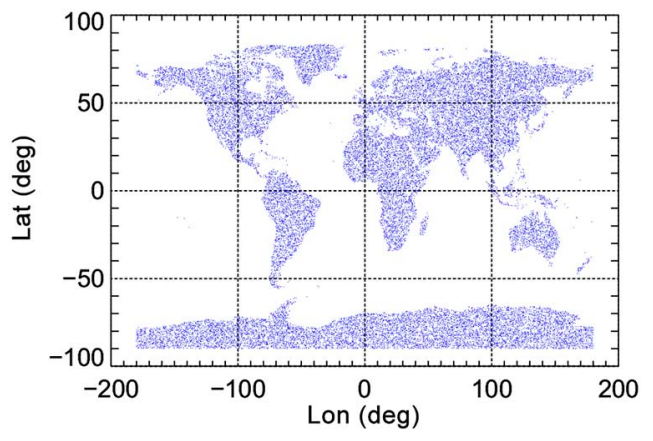

Fig. 10. Local distribution of target coordinates used to simulate 20000 statistical acquisitions. Each point represents a scene center coordinate.

commanding algorithms are invoked. During the calculations, the commanding parameters derived in the mode design and optimization phase (cf. Section II) are used as input. The reference orbit position of the satellite, its attitude, and accurate DEM information of the scene are considered. These parameters significantly differ, depending on the scenes location on the Earth. Therefore, a global simulation approach is required to obtain globally valid results. The output of the commanding algorithms is a set of parameters describing the acquisition in terms of radar commanding parameters, such as the PRF, the azimuth antenna steering rate, and others. Additionally, output messages are created giving information about timing problems, scene size degradations, or even failed results. The last stage in the global performance assessment workflow is to evaluate the output messages and to estimate the performance of each simulated scene based on the calculated radar commanding parameters. AASR, RASR, the range scene size, and the acquisition duration are analyzed as shown in Section III-B because these parameters are the most challenging ones for the TerraSAR-X staring spotlight mode and significantly vary with the actual acquisition geometry. However, the global performance assessment approach as described here is not limited to these aspects and parameters. For other modes, other parameters can be analyzed, depending on the specific challenges faced by the mode [2].
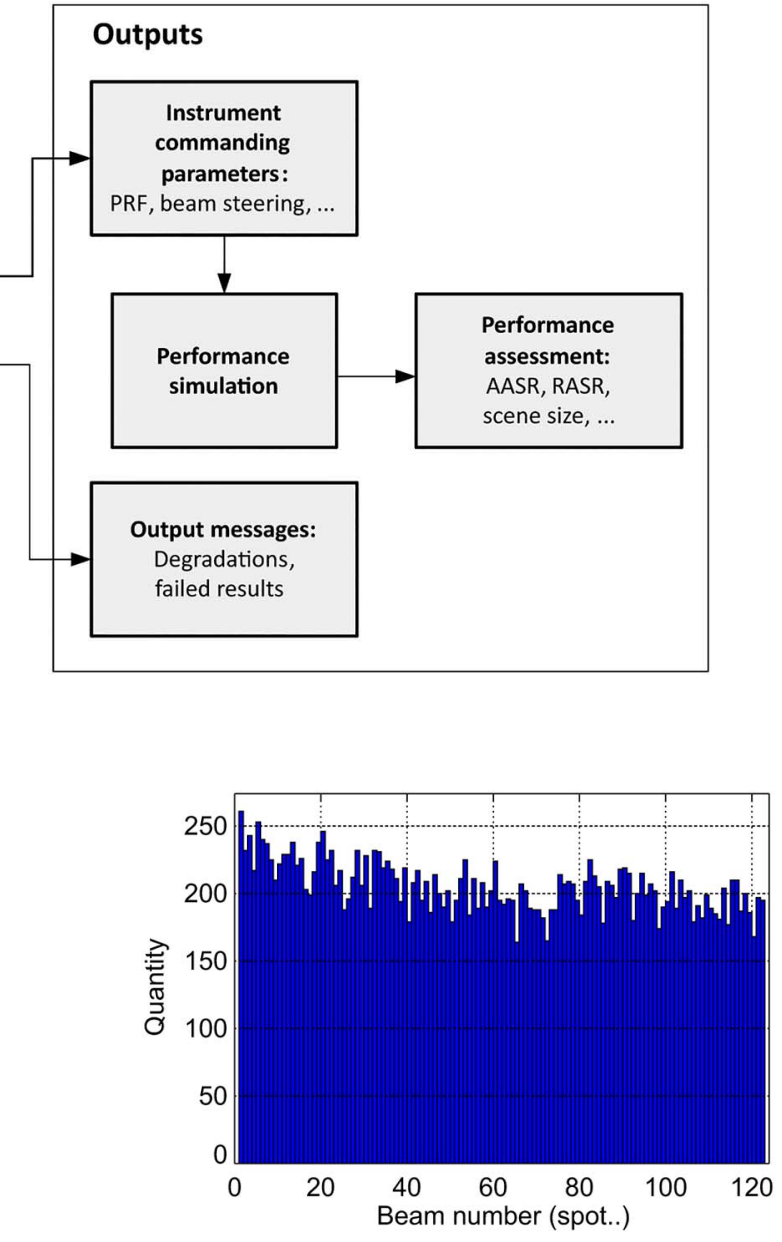

Fig. 11. Distribution of the elevation beams used to simulate 20000 statistical acquisitions. The first beam spot_001 has an incidence angle of $15^{\circ}$, whereas the last beam spot_122 has an incidence angle of $60^{\circ}$.

\section{B. Global Simulation Results}

A very promising first result of the simulation campaign was that all acquisitions could be generated by the operational software without any problems, i.e., there was no failed result. For every scene, the timing parameters could be successfully calculated. Even for scenes with challenging timing conditions due to difficult terrain with steep slopes, no problem occurred. Thirteen of these acquisitions over difficult terrain were commanded on the satellite for validation purposes. A quality evaluation of the image products revealed no peculiarities. The ASRs are calculated for several target positions distributed over range and azimuth for all simulated scenes. The operational commanding parameters are used as inputs. Fig. 12 shows the AASR for two characteristic target positions within a staring spotlight scene. On the left, the values for a point target at azimuth scene center are given, representing the best-case AASR within each scene. The results are depicted as the contour lines of a 2-D histogram of AASR and incidence angle. As the beam distribution is approximately uniform (cf. Fig. 11), the histogram reflects the AASR distribution, highlighting the dominant AASR region for a given incidence angle. On the right, the worst-case AASR for each scene is shown, which corresponds to a point target at the azimuth edge of the scene. 


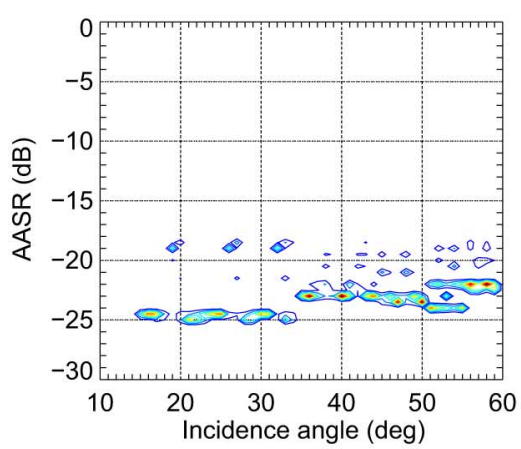

(a)
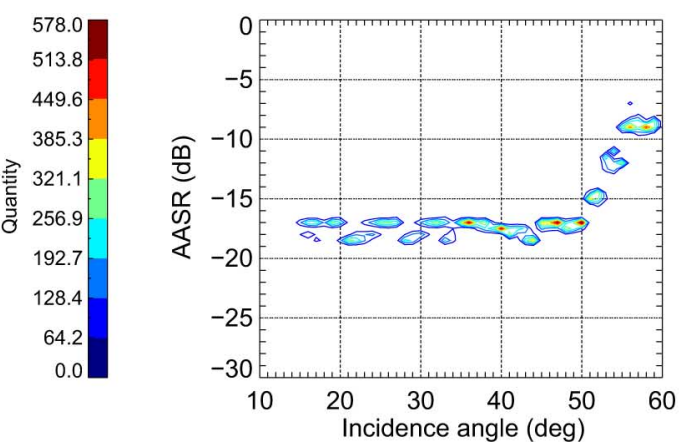

(b)

Fig. 12. Contour lines of the two-dimensional (2-D) histogram of the AASR for (a) target at scene center and (b) azimuth edge of the scene of each simulated acquisition.

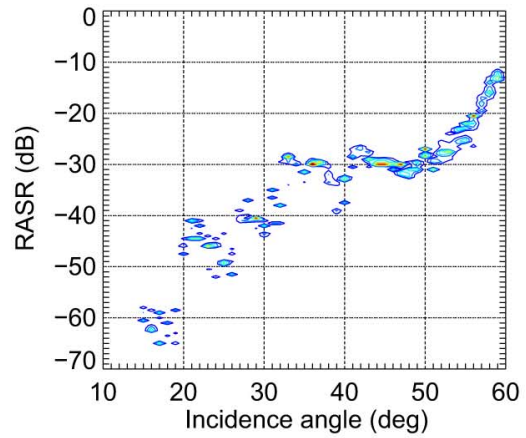

(a)
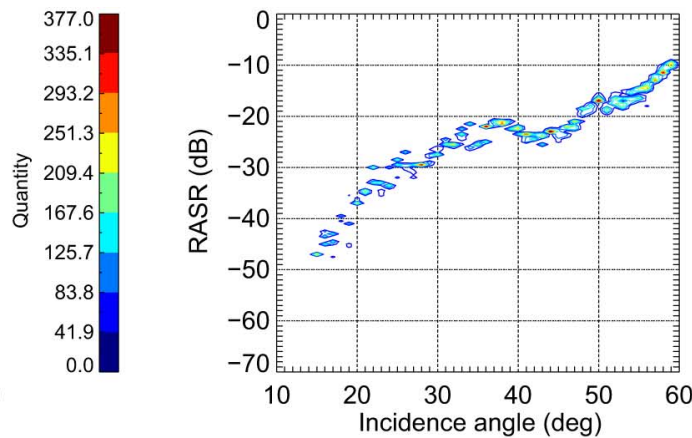

(b)

Fig. 13. Contour lines of the 2-D histogram of the RASR for (a) best target and (b) worst target of each simulated acquisition.

The edge point targets for this simulation have been selected according to the azimuth scene size definition described in Section II-C. The AASR performance for a specific target location (e.g., scene center) within a set of scenes acquired with the same elevation beam and therefore incidence angle is different, since the PRF for each acquisition is individually selected by the operational commanding algorithms. The selection depends on the specific timing conditions reflecting the orbit altitude and the scene topography. This results in a broadening of the histogram in ordinate direction for a given incidence angle. This effect can be observed especially for scene center targets [Fig. 12(a)] above $35^{\circ}$ incidence angle. Additionally, a stronger AASR variation at a fixed incidence angle can be observed for scene center targets compared to scene edge targets. This is due to a larger AASR gradient for scene center targets, if the target PRF (indicated by red arrows in Fig. 4) can not be selected due to timing constraints. Obviously, for elevation beams with approximately $19^{\circ}, 27^{\circ}$, and $33^{\circ}$ incidence angle, a PRF further away from the target PRF has to be selected at least for a significant amount of the acquisitions [Fig. 12(a)]. However, the AASR for these acquisitions is more homogeneous over the azimuth scene extent. The best case AASR is in the order of $-19 \mathrm{~dB}$, whereas the worst-case AASR is around $-17 \mathrm{~dB}$ for acquisitions with these incidence angles. Overall, the AASR performance is better than $-17 \mathrm{~dB}$ for all point targets within the scene for incidence angles up to $50^{\circ}$.

Similar to the AASR characteristics, the RASR performance has been evaluated. The results for the best-case target locations within the scenes are shown in Fig. 13 on the left and for the worst-case target locations on the right. Overall, the RASR performance is deteriorating with increasing incidence angle. This trend is clearly observable, although the target PRF is lowered with increasing incidence angle to counteract rising ambiguity powers (cf. Fig. 5). Compared to the AASR performance, slight changes of the PRF away from the target PRF do not lead to significantly changed RASR values. The histograms are compact in RASR direction especially for values above $-30 \mathrm{~dB}$. This can be explained by smaller gradients of RASR (Fig. 3) compared to the AASR values (Fig. 4) as function of the PRF. For incidence angles up to $47^{\circ}$, an RASR performance of $20 \mathrm{~dB}$ or better is achieved for all target locations within a scene.

Besides ambiguity levels, the range swath width is analyzed based on the global simulation results. The scene size is limited by instrument constraints, namely the data rate and the echo buffer size. The possible length of the receive echo window depends on the sampling rate and therefore the used range chirp bandwidth and on the PRF. For the staring spotlight mode, the maximum possible range chirp bandwidth of $300 \mathrm{MHz}$ is used to achieve the best possible range resolution. The scene size varies with the used PRF which depends on the individual acquisition scenario. The 2-D histogram of the ground range scene size depending on the incidence angle is depicted in Fig. 14. The overall trend of decreasing scene sizes with increasing incidence angle is caused by the slant-to-ground range projection. However, changes in the used PRF range over incidence angle are clearly visible, e.g., around $34^{\circ}$ incidence angle. Here, the target PRF changes from 5100 to $4450 \mathrm{~Hz}$ 

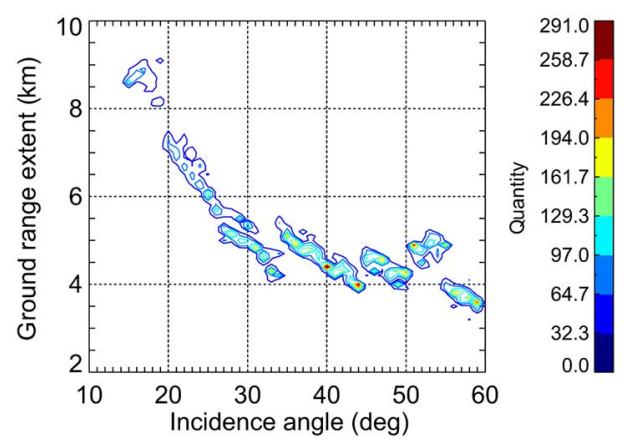

Fig. 14. Contour lines of the 2-D histogram of all simulated nominal ground range scene extents.

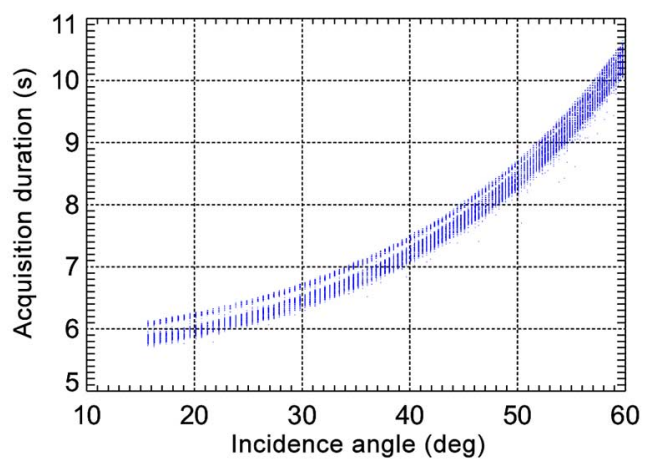

Fig. 15. Scatter plot of the net duration of all simulated staring spotlight acquisitions as a function of incidence angle. Calibration and noise pulse recording before start and after the end of the acquisition further prolongate the acquisition for about $1 \mathrm{~s}$.

allowing for a positive step in the ground range scene size, although the incidence angle increases.

The azimuth scene size is not analyzed here since it does not depend on the actual PRF of the acquisition but only on the specifications derived in Section II-C, which are based on the predicted AASR and NESZ performance.

Another interesting acquisition parameter is the duration. All spotlight modes are innately noncontinuous in azimuth. The longer the acquisition lasts, the larger is the gap between two consecutive acquisitions. For the constant azimuth steering span of $4.42^{\circ}$ used for staring spotlight, the acquisition duration depends on the satellite altitude and the incidence angle. The duration increases with incidence angle and orbit altitude. Fig. 15 depicts the net acquisition duration, i.e., only imaging pulses, versus incidence angle as a scatter plot for all simulated scenes. For the complete execution time of a staring spotlight acquisition, the times required to record calibration and noise measurements add about one additional second. The variation of approximately $0.5 \mathrm{~s}$ in the net acquisition duration for a fixed incidence angle is due to the orbit altitude variation. The apparent white band in the upper part of the scattered area-especially visible for low incidence angles-appears due to the absence of landmasses and therefore simulated acquisitions with orbit altitudes between the south of Patagonia and north of the Antarctic Peninsula.

\section{Evaluation of Staring Spotlight Acquisitions}

In order to complement and verify the simulations shown in the previous sections and to complete the TerraSAR-X staring

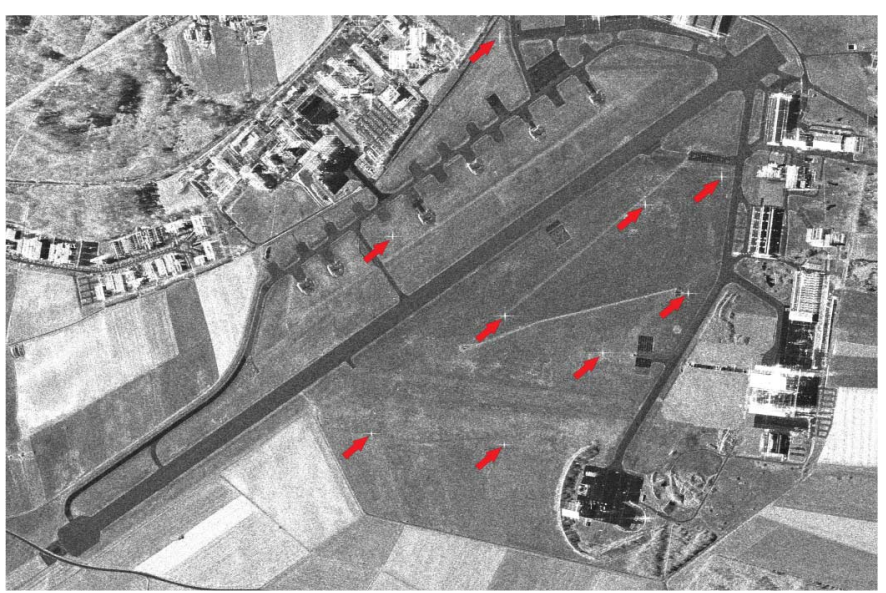

Fig. 16. Zoom into a TerraSAR-X staring spotlight scene over the DLR Oberpfaffenhofen SAR calibration facility showing nine corner reflectors marked with red arrows. For the acquisition elevation, beam spot_010 with $20^{\circ}$ incidence angle was used.

TABLE III

IRF CHARACTERISTICS OF 27 POINT TARgET ANALYSES

\begin{tabular}{|l|l|c|c|}
\hline Irf parameter & Median & Min & Max \\
\hline Azimuth resolution $(\mathrm{m})$ & 0.219 & 0.218 & 0.223 \\
\hline Slant range resolution $(\mathrm{m})$ & 0.604 & 0.600 & 0.607 \\
\hline Azimuth PSLR (dB) & -27.7 & -31.8 & -21.3 \\
\hline Range PSLR (dB) & -31.0 & -31.6 & -29.2 \\
\hline Azimuth ISLR (dB) & -19.1 & -20.2 & -17.1 \\
\hline Range ISLR (dB) & -19.7 & -19.9 & -19.4 \\
\hline
\end{tabular}

spotlight mode specifications, several acquisitions over selected locations have been acquired. These were areas with deployed corner reflectors for the analysis of the IRF and areas containing calm water bodies to estimate the NESZ characteristics. Agricultural fields have been acquired to evaluate the radiometric resolution. The data were processed with an updated version of the TerraSAR-X multimode SAR processor [24], capable of handling staring spotlight data. The same processor is used for the operational staring spotlight processing [25].

\section{A. Point Target Analysis}

The IRF characteristics have been analyzed using single look, slant range, complex data of three acquisitions acquired over the Oberpfaffenhofen SAR calibration facility where nine corner reflectors had been deployed. Fig. 16 shows a zoom into one of the image products acquired with the elevation beam spot_010 with an incidence angle of $20^{\circ}$. All nine corner reflectors appear as bright point targets on grassland as background. From their responses, the resolutions in range and azimuth, as well as the integrated sidelobe ratio (ISLR) and the peak-toside lobe ratio (PSLR) in both directions, can be determined. Three acquisitions with steep $\left(20^{\circ}\right)$, medium $\left(47^{\circ}\right)$, and shallow $\left(59^{\circ}\right)$ incidence angle have been analyzed. The results are shown in Table III. The resolutions agree very well with the theoretical predictions. However, the achieved azimuth focusing quality depends on the reference height used by the processor as shown in [25]. In order to account for reference DEM errors, the specification of the azimuth resolution is lowered to $24 \mathrm{~cm}$ 

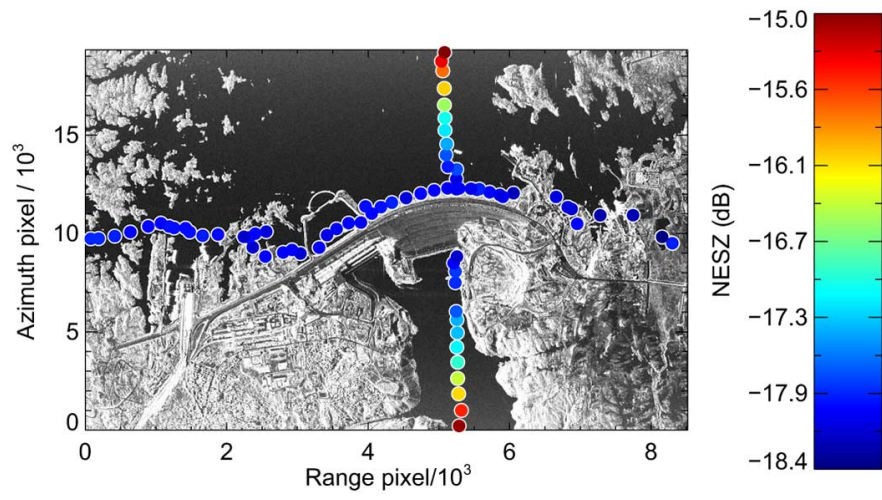

Fig. 17. NESZ evaluated by a distributed target analysis of a full staring spotlight image over the Aswan dam, Egypt. The scene size is approximately $5.0 \mathrm{~km}$ in range and $3.2 \mathrm{~km}$ in azimuth direction. The regions defined as distributed targets are indicated as colored dots. The color represents the NESZ-see color bar. The used beam (spot_081) has an incidence angle of $50^{\circ}$.

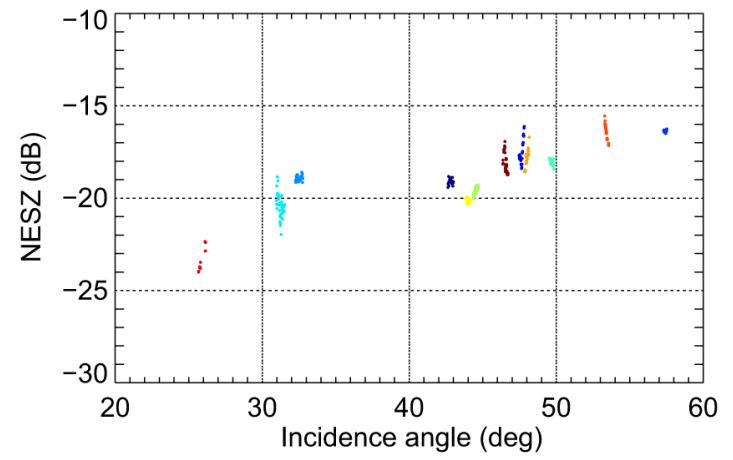

Fig. 18. NESZ derived from low backscatter area measurements over water bodies for different beams. The areas have been analyzed over the whole range of the scene at the center in azimuth direction. Each color represents measurements from a different acquisition.

(cf. Table V). The median values for PSLR and ISLR are below -25 and $-18 \mathrm{~dB}$, respectively. These values correspond to the specifications of the high-resolution spotlight mode. However, the maximum measured values for staring spotlight are worse for azimuth. This can be explained by the extremely long integration times. Even very small disturbances during the synthetic aperture time of up to $10.7 \mathrm{~s}$ can lead to a degradation of the IRF. For this reason, the specification of PSLR and ISLR in the azimuth direction for staring spotlight is relaxed to -20 and $-16 \mathrm{~dB}$, respectively (cf. Table V).

\section{B. NESZ Estimation}

To assess the NESZ performance, acquisitions over calm water bodies (lakes, rivers, and coastal regions) have been commanded. Fig. 17 shows a staring spotlight acquisition of the Aswan dam in Egypt with the river Nile as an example. Colored dots represent areas over calm water that have been selected as distributed targets. The targets are lying approximately on two lines, resulting in an elevation and an azimuth cut. The colors represent the estimated NESZ according to the color bar. It ranges from $-18.5 \mathrm{~dB}$ at scene center to $-15 \mathrm{~dB}$ at the azimuth edges of the scene. The used elevation beam is spot_081 with an incidence angle of $50^{\circ}$. The measured
TABLE IV

RESOLUtions IN GROUND RANGe $\left(\delta_{\mathrm{Rg}}\right)$ AND AZIMUth $\left(\delta_{\mathrm{Az}}\right)$ FOR High-Resolution Spotlight Spatially EnHanced (SE) AND Staring SPOTLight Radiometricly EnHANCEd (RE) Products For SiX ANALYZed IMAges ACQuired With Three DifFERENT ELEVATION BEAMS

\begin{tabular}{|l|l|l|l|l|}
\hline & \multicolumn{4}{|l|}{ Staring spotlight RE } \\
\hline Beam & $\delta_{\mathrm{Rg}}(\mathrm{m})$ & $\delta_{\mathrm{Az}}(\mathrm{m})$ & ENL $_{\text {proc. }}$ & ENL $_{\text {ROI }}$ \\
\hline spot_013 & 1.60 & 1.35 & 6.52 & 6.17 \\
\hline spot_042 & 1.04 & 1.04 & 5.11 & 4.64 \\
\hline spot_055 & 0.98 & 0.98 & 5.27 & 4.19 \\
\hline & \multicolumn{4}{|l|}{ High-resolution spotlight SE } \\
\hline Beam & $\delta_{\mathrm{Rg}}(\mathrm{m})$ & $\delta_{\mathrm{Az}}(\mathrm{m})$ & ENL $_{\text {proc. }}$ & ENL \\
\hline spot_013 & 1.63 & 1.37 & 1.30 & 1.27 \\
\hline spot_042 & 1.03 & 1.16 & 1.02 & 1.02 \\
\hline spot_055 & 1.00 & 1.16 & 1.15 & 1.09 \\
\hline
\end{tabular}

The resolutions as well as the equivalent number of looks (ENL) ENL proc. are annotated in the products by the operational processor. The ENL estimated by a distributed target analysis over a region of interest (ROI) $\mathrm{ENL}_{\mathrm{ROI}}$ is shown in the last column.

TABLE V

Specified Parameters of The TerraSAR-X Staring SPotlight Mode for the Full Performance Range Over Ground Range (RG) AND AZIMUTH (AZ)

\begin{tabular}{|ll|l|l|}
\hline Parameter & & $\begin{array}{l}\text { Full performance } \\
\text { range }\end{array}$ & $\begin{array}{l}\text { Data collection } \\
\text { range }\end{array}$ \\
\hline Incidence angle $\left({ }^{\circ}\right)$ & $20, \ldots, 45$ & $15, \ldots, 60$ \\
\hline Geometric & $\mathrm{Rg}$ & $1.77, \ldots, 0.85$ & $2.35, \ldots, 0.70$ \\
resolution $(\mathrm{m})$ & $\mathrm{Az}$ & 0.24 & 0.24 \\
\hline Scene size $(\mathrm{km})$ & $\mathrm{Rg}$ & $7.5, \ldots, 4.6$ & $9.0, \ldots, 3.5$ \\
& $\mathrm{Az}$ & $2.5, \ldots, 2.8$ & $2.5, \ldots, 3.7$ \\
\hline RASR $(\mathrm{dB})$ & & -20 & -10 \\
\hline AASR $(\mathrm{dB})$ & & -17 & -9 \\
\hline NESZ $(\mathrm{dB})$ & & -18 & -16 \\
\hline PSLR $(\mathrm{dB})$ & $\mathrm{Rg}$ & -25 & -25 \\
& $\mathrm{Az}$ & -20 & -20 \\
\hline ISLR (dB) & $\mathrm{Rg}$ & -18 & -18 \\
& $\mathrm{Az}$ & -16 & -16 \\
\hline
\end{tabular}

For the data collection range, the worst-case values based on simulation results and measurements are annotated.

NESZ variation over azimuth of $3.4 \mathrm{~dB}$ corresponds very well to the simulation results presented in [5] (for the used elevation beam with $50^{\circ}$ incidence angle, $75 \%$ of the boresight azimuth beam width are used). The shape of the azimuth NESZ profile as predicted by the simulation is clearly visible. The profile around the azimuth scene center in range direction is almost flat, with NESZ values below -18 dB. Generally, the NESZ profile in range direction corresponds to the shape of the elevation antenna pattern. In the shown example, only a small fraction of the elevation antenna pattern is used at the incidence angle of $50^{\circ}$. Therefore, the NESZ variation over range is smaller than the $6 \mathrm{~dB}$ that one could expect for using the full 3-dB elevation beam width.

The same NESZ measurement approach based on low backscatter, calm water areas was employed to evaluate several scenes acquired with different elevation beams. In Fig. 18, the NESZ profiles over range (at azimuth scene center) of 12 acquisitions are depicted over incidence angle. The measurements confirm an NESZ better than $-18 \mathrm{~dB}$ for an incidence angle range up to $45^{\circ}$. This value of $-18 \mathrm{~dB}$ is specified for the full performance range of the staring spotlight mode (cf. 

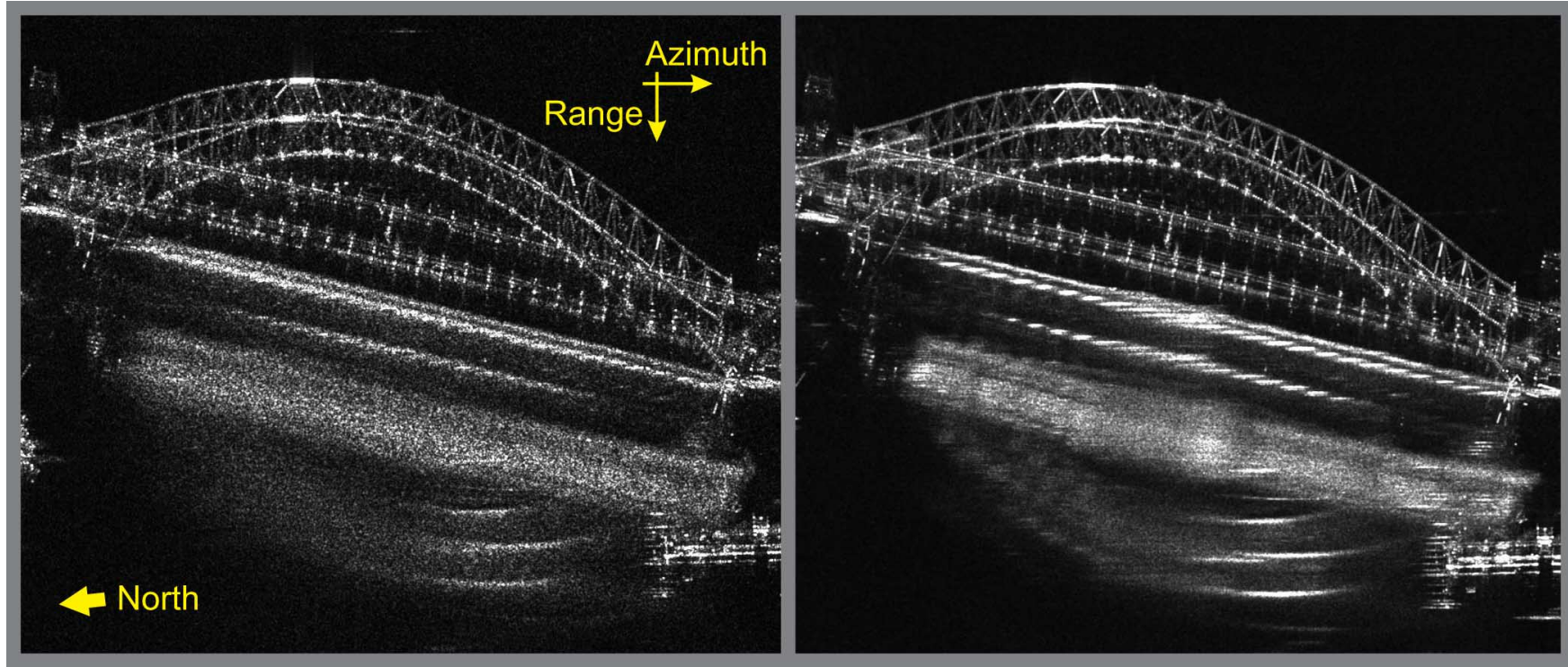

Fig. 19. Zoom into SAR images of Sydney, Australia (Harbour Bridge) acquired in high-resolution spotlight mode (left) and in staring spotlight mode (right). The used elevation beam spot_048 has an incidence angle of $38^{\circ}$. Both images are processed to the same resolution resulting in a higher number of looks for the staring spotlight image.

Table $\mathrm{V}$ ) for the center of the scene in azimuth direction. This NESZ degrades toward the azimuth edges of the scene.

\section{Radiometric Resolution}

In order to evaluate the radiometric resolution based on the equivalent number of looks (ENL) of the TerraSAR-X staring spotlight mode, three acquisitions are analyzed. The elevation beams of the acquisitions are spot_013, spot_042, and spot_055 with $22^{\circ}, 36^{\circ}$, and $41^{\circ}$ incidence angle, respectively. The acquisitions are processed to multilook ground-range detected products in the radiometrically enhanced (RE) variant, averaging approximately five to seven looks [1]. For comparison reasons, the same scenes have also been acquired using the high-resolution spotlight mode. These acquisitions are processed to spatially enhanced (SE) products. The SE product is processed to the best possible square ground resolution [1]. These product variants are chosen since their spatial resolutions are very similar to each other. In Table IV, the values annotated by the operational processor for the spatial resolutions in range $\left(\delta_{\mathrm{Rg}}\right)$ and azimuth $\left(\delta_{\mathrm{Az}}\right)$ as well as the $\mathrm{ENL}_{\text {proc. }}$ are summarized. The staring spotlight products have approximately five times the number of looks than the high-resolution spotlight products if processed to the same spatial resolution.

In order to confirm the annotated number of looks, the data were analyzed using a distributed target technique. The ENL can be estimate from the statistics of the acquired data over an ROI according to

$$
\mathrm{ENL}_{\mathrm{ROI}}=\left(\frac{\mu_{\mathrm{ROI}}}{\sigma_{\mathrm{ROI}}}\right)^{2} .
$$

The parameters $\mu_{\mathrm{ROI}}$ and $\sigma_{\mathrm{ROI}}$ represent the mean and the standard deviation of the image pixel intensity (power), respectively [11], [26]. The ROI is selected to be a homogeneous backscatter area, e.g., an agricultural field, treated as a distributed target [19], [27]. In Table IV, the radiometric resolutions derived by this method are shown as $\mathrm{ENL}_{\mathrm{ROI}}$ for the six analyzed scenes. Each of the values represents the median over several ROIs selected in every scene.

The number of looks annotated by the processor $\left(\mathrm{ENL}_{\mathrm{proc}}\right)$ is in all cases slightly larger than the one derived by the distributed target analysis $\left(\mathrm{ENL}_{\mathrm{ROI}}\right)$, but the results agree very well. The reason for the differences might be a residual texture component in the analyzed ROIs, e.g., agricultural fields. The areas are visually chosen to be without any texture and homogeneous. Nevertheless, some structure might be present.

\section{First ACQUisitions From Operational ENVIRONMENT}

The main difference between the high-resolution spotlight and staring spotlight mode is the significantly larger azimuth steering angle of the latter mode. In Fig. 19, exemplary acquisitions of both modes are compared. A zoom into the multilook ground-range detected TerraSAR-X products shows a part of Sydney, Australia (the Harbour Bridge area). The left image is derived from a high-resolution spotlight acquisition, whereas the right image shows details of a staring spotlight image. Both acquisitions are acquired with a range chirp bandwidth of $300 \mathrm{MHz}$ and processed to the same azimuth resolution. Therefore, the pixel spacing and the resolution are identical. However, the staring spotlight image has a better radiometric performance since a mulitlooking factor of approximately five could be applied. The reduced Speckle noise facilitates the identification of single elements, e.g., of the arch's metal structure. Additionally, the longer synthetic aperture and the larger angular azimuth steering span are visible as the bright segment on the upper left part of the arch structure is much longer in the staring spotlight acquisition than in the high-resolution spotlight acquisition. With staring spotlight, a larger part of the 

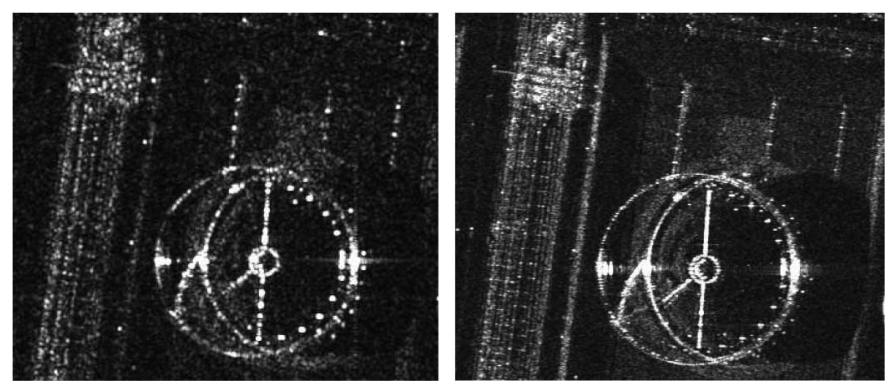

Fig. 20. Tanks of an oil refinery in Rotterdam, the Netherlands, acquired with elevation beam spot_075 at an incidence angle of $48^{\circ}$. The high-resolution spotlight acquisition (left) was acquired in 2009. The staring spotlight acquisition (right) was acquired in 2013.

curved structure is imaged at an azimuth angle, which causes a strong reflection of the incident electromagnetic wave.

Fig. 20 shows another pair of high-resolution spotlight (left) and staring spotlight (right) images. The high-resolution spotlight image has already been acquired in 2009 by the TerraSAR-X satellite, whereas the staring spotlight image has been acquired in 2013 by the TanDEM-X satellite. The images are a zoom into the area of a refinery in Rotterdam, the Netherlands, and show a tank and pipelines. Both images are processed to the same geometric resolution. Therefore, the radiometric performance of the staring spotlight image is improved by multilooking. Especially, the pipelines in the upper part of the images and the structure of the ground surrounding the tank are better visible. Also, the shadow of the tank on the right side of the images is more homogeneous in the staring spotlight image.

Both images are examples for the improvements achieved by the operationalization of the staring spotlight mode regarding the monitoring capabilities for critical infrastructure with the TerraSAR-X system.

\section{CONCLUSION}

This paper summarizes the optimizations conducted during the operationalization of the TerraSAR-X staring spotlight mode. The challenges faced by the increased azimuth antenna steering-beyond the limits the system had originally been designed for-have been addressed. Operational commanding parameters such as PRF ranges, margin settings, and scene size definitions have been determined. A novel global performance assessment approach was introduced. This approach utilizes the operational command generation software embedded in a simulation framework. Globally distributed acquisitions are simulated and the results evaluated. This approach delivers performance estimates as close to the real-world system as possible. The global simulation demonstrated the suitability of the commanding parameters for acquisitions all over the world with changing orbit altitudes and challenging topographic conditions. On top of that, the simulation approach is part of the verification process for the updated operational command generation algorithms capable of handling the staring spotlight mode. Finally, measurement results of IRF parameters and NESZ characteristics have been presented and image examples showed an increase in target details and detectability provided by of the operational staring spotlight products.

An essential output of the mode operationalization phase is the specification of the image products. The analysis described in this paper in hand delivered the inputs for an updated version of the TerraSAR-X basic product specification [1]. A summary of the specified performance parameters of the TerraSAR-X staring spotlight mode for the full performance incidence angle range is given in Table V. Additionally, worst-case parameters for the data collection incidence angle range are provided. The azimuth scene size definition is based on results from Section II. In Section III, the range scene size, as well as RASR and AASR, has been derived. The specification of the geometric resolution, the NESZ, and the sidelobe ratios is based on results shown in Section IV.

\section{REFERENCES}

[1] T. Fritz and M. Eineder, "TerraSAR-X basic product specification document," German Aerospace Center (DLR), Oberpfaffenhofen, Germany, Tech. Rep. 1.9, 2013 [Online]. Available: http://terrasar-x.dlr.de/pdfs/TXGS-DD-3302_1.9.pdf

[2] T. Kraus et al., "A global performance assessment approach for the TerraSAR-X staring spotlight and wide ScanSAR modes," in Proc. Eur. Conf. Synth. Aperture Radar (EUSAR), Berlin, Germany, 2014, pp. 997-1000.

[3] W. G. Carrara, R. S. Goodman, and R. M. Majewski, Spotlight Synthetic Aperture Radar. Norwood, MA, USA: Artech House, 1995.

[4] R. Mailloux, Phased Array Antenna Handbook. Norwood, MA, USA: Artech House, 2005.

[5] J. Mittermayer, S. Wollstadt, P. Prats, and R. Scheiber, "The TerraSAR$\mathrm{X}$ staring spotlight mode concept," IEEE Trans. Geosci. Remote Sens., vol. 52, no. 6, pp. 3695-3706, Jun. 2014.

[6] J. Mittermayer, S. Wollstadt, P. Prats, R. Scheiber, and W. Koppe, "Staring spotlight imaging with TerraSAR-X," in Proc. IEEE Int. Geosci. Remote Sens. Symp. (IGARSS), Munich, Germany, 2012, pp. 1606-1609.

[7] M. Eineder, N. Adam, R. Bamler, N. Yague-Martinez, and H. Breit, "Spaceborne spotlight SAR interferometry with TerraSAR-X," IEEE Trans. Geosci. Remote Sens., vol. 47, no. 5, pp. 1524-1535, May 2009.

[8] U. Steinbrecher, D. Schulze, J. Böer, and J. Mittermayer, "TerraSAR-X instrument operations rooted in the system engineering and calibration project," IEEE Trans. Geosci. Remote Sens., vol. 48, no. 2, pp. 633-641, Feb. 2010.

[9] P. Prats et al., "High precision SAR focusing of TerraSAR-X experimental staring spotlight data," in Proc. IEEE Int. Geosci. Remote Sens. Symp. (IGARSS), Munich, Germany, 2012, pp. 3576-3579.

[10] P. Prats-Iraola et al., "On the processing of very high-resolution spaceborne SAR data," IEEE Trans. Geosci. Remote Sens., vol. 52, no. 10, pp. 6003-6016, Oct. 2014.

[11] I. Cumming and F. Wong, Digital Signal Processing of Synthetic Aperture Radar Data: Algorithms and Implementation. Norwood, MA, USA: Artech House, 2005.

[12] F. J. Harris, "On the use of windows for harmonic analysis with the discrete Fourier transform," Proc. IEEE, vol. 66, no. 1, pp. 51-83, Jan. 1978.

[13] S. D' Amico, C. Arbinger, M. Kirschner, and S. Campagnola, "Generation of an optimum target trajectory for the TerraSAR-X repeat observation satellite," in Proc. 18th Int. Symp. Space Flight Dyn., Munich, Germany, 2004, pp. 1-6.

[14] H. Fiedler, E. Boerner, J. Mittermayer, and G. Krieger, "Total zero Doppler steering-A new method for minimizing the Doppler centroid," IEEE Geosci. Remote Sens. Lett., vol. 2, no. 2, pp. 141-145, Apr. 2005.

[15] M. Bachmann, M. Schwerdt, and B. Bräutigam, "TerraSAR-X antenna calibration and monitoring based on a precise antenna model," IEEE Trans. Geosci. Remote Sens., vol. 48, no. 2, pp. 690-701, Feb. 2010.

[16] T. Kraus, B. Bräutigam, C. Grigorov, J. Mittermayer, and S. Wollstadt, "TerraSAR-X staring spotlight mode optimization," in Proc. Asia-Pac. Conf. Synth. Aperture Radar (APSAR), Tsukuba, Japan, 2013, pp. 16-19.

[17] F. Ulaby and C. Dobson, Handbook of Radar Scattering: Statistics for Terrain. Norwood, MA, USA: Artech House, 1989. 
[18] J. Curlander and R. McDonough, Synthetic Aperture Radar: Systems and Signal Processing. Hoboken, NJ, USA: Wiley, 1991.

[19] J. Mittermayer et al., "TerraSAR-X system performance characterization and verification," IEEE Trans. Geosci. Remote Sens., vol. 48, no. 2, pp. 660-676, Feb. 2010.

[20] R. Kahle et al., "First in-orbit experience of TerraSAR-X flight dynamics operations," in Proc. 20th Int. Symp. Space Flight Dyn., Annapolis, MD, USA, 2007, pp. 1-11.

[21] M. Werner, "Shuttle radar topography mission (SRTM): Mission overview," J. Telecommun. (Freq.), vol. 55, no. 3/4, pp. 75-79, 2001.

[22] E. Rodriguez, C. S. Morris, and E. J. Belz, "A global assessment of the SRTM performance," Photogramm. Eng. Remote Sens., vol. 72, no. 3, pp. 249-260, 2006.

[23] D. A. Hastings and P. K. Dunbar, "Development \& assessment of the global land one-km base elevation digital elevation model (GLOBE)," in Proc. ISPRS Comm. IV Symp. GIS Between Vis. Appl., Stuttgart, Germany, 1998, vol. 32, no. 4, pp. 218-221.

[24] H. Breit et al., "TerraSAR-X SAR processing and products," IEEE Trans. Geosci. Remote Sens., vol. 48, no. 2, pp. 727-740, Feb. 2010.

[25] H. Breit, M. Fischer, U. Balss, and T. Fritz, "TerraSAR-X staring spotlight processing and products," in Proc. Eur. Conf. Synth. Aperture Radar (EUSAR), Berlin, Germany, 2014, pp. 193-196.

[26] P. Olivier and D. Vidal-Madjar, "Empirical estimation of the ERS-1 SAR radiometric resolution," Int. J. Remote Sens., vol. 15, no. 5, pp. 11091114, 1994.

[27] M. Schwerdt, M. Zink, and D. Hounam, "A treatment of SAR radiometric resolution including geolocated ambiguous energy," in Proc. CEOS-SAR Workshop, Tokyo, Japan, 2001, pp. 1-4.

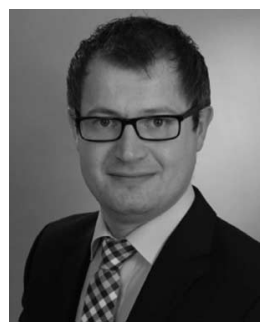

Thomas Kraus received the M.Sc. degree in electrical engineering from the University of Ulm, Ulm, Germany, in 2009.

In 2010, he joined the Microwaves and Radar Institute, German Aerospace Center (DLR), Oberpfaffenhofen, Germany. He is working in the field of spaceborne synthetic aperture radar (SAR) instrument commanding and analysis of scientific and experimental acquisitions in the framework of the projects TerraSAR-X and TanDEM-X. His research interests include radar system performance and the development of innovative SAR modes.

Mr. Kraus was awarded with the CASSIDIAN (Airbus) ARGUS Award 2010 for his master thesis and contributed to a project which received the EEEfCOM Innovation Award 2010.

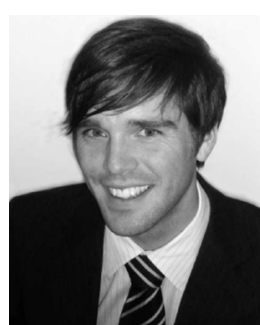

Benjamin Bräutigam received the Dipl.-Ing. and Dr.-Ing. (Ph.D.) degrees in electrical engineering from Karlsruhe Institute of Technology (University of Karlsruhe), Karlsruhe, Germany, in 2003 and 2014, respectively.

In 2003, he was a Visiting Scientist at the NOAA Environmental Technology Laboratory, Boulder, $\mathrm{CO}$, USA. In 2004, he joined the Microwaves and Radar Institute, German Aerospace Center (DLR), Oberpfaffenhofen, Germany. He was responsible for instrument calibration of spaceborne synthetic aperture radar (SAR) projects like TerraSAR-X, TanDEM-X, and Sentinel-1. Since 2008, he has been the Head of the Research Group for system performance working on actual and future SAR satellite missions. His research interests include calibration and performance analysis of radar systems.

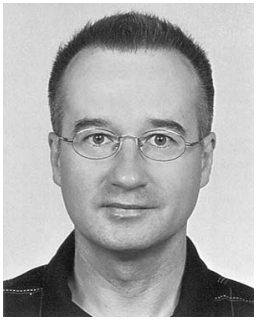

Josef Mittermayer was born in Wartenberg, Oberbayern, Germany, in 1967. He received the Diploma degree in electrical engineering from the Technical University of Munich, Munich, Germany, in 1995, the M.S. degree in space system engineering from the Delft University of Technology, Delft, The Netherlands, in 2004, and the Ph.D. degree in spotlight-SAR processing from the University of Siegen, Siegen, Germany, in 2000.

From 1994 to 2001, he was with the Signal Processing Group, Microwaves and Radar Institute, German Aerospace Center (DLR), Oberpfaffenhofen, Germany. From 2002 to 2008, he was working in the TerraSAR-X project with DLR. From January 2004 until the end of the commissioning phase in 2008, he was the Group Leader and the Project Manager of System Engineering and Calibration, one of the three subprojects that form the TerraSAR-X Ground Segment. In addition, he was technically responsible for the TerraSAR-X commissioning phase. $\mathrm{He}$ is currently as Scientist with the Microwaves and Radar Institute, working in the field of SAR system engineering, SAR processing and the development of new SAR-modes among others bi-directional SAR and improved starring spotlight modes.

Dr. Mittermayer and his colleagues were recipients of the IEEE Geoscience and Remote Sensing Society Transactions Prize Paper Award for a paper on air and spaceborne stripmap and ScanSAR processing in 1996. He was a recipient of the DLR Science Award for his work on spotlight-SAR processing and the DLR Forschungssemester for his contribution to the TerraSAR-X project, in 2011.

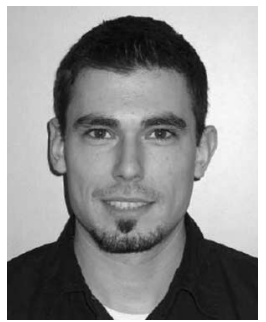

Steffen Wollstadt received the Dipl.-Ing. (M.Sc.) degree in electrical engineering from the Technical University of Darmstadt, Darmstadt, Germany, in 2005 .

In 2006, he joined the Microwaves and Radar Institute, German Aerospace Center (DLR), Oberpfaffenhofen, Germany. From 2006 to 2008, he was with the Satellite SAR Systems Department as Project Engineer in the System Engineering and Calibration part of the TerraSAR-X ground segment, where he worked on the TerraSAR-X and TanDEM-X instrument command generation. Since 2009, he has been with the Radar Concepts Department, Microwaves and Radar Institute in the SAR Missions Research Group, where he was working on TOPSAR Sentinel-1 image quality and SAR performance investigations and on experiments with TerraSAR-X and TanDEM-X about novel imaging modes implying also interferometric problems. Currently, he is working on a spaceborne SAR mission study, which uses along-track interferometry for the measurement of ocean surface currents.

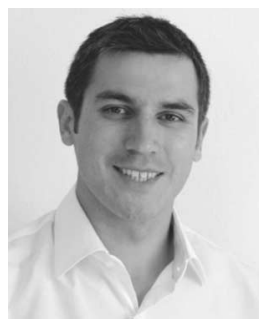

Christo Grigorov received the M.Sc. degree in computer science from the Technical University Sofia, Sofia, Bulgaria, in 2005, and the double M.Sc. degrees in space science and technology from JuliusMaximilians University of Wuerzburg, Wuerzburg, Germany, and from Lulea University of Technology, Lulea, Sweden, in 2007.

In 2008, he joined the Microwaves and Radar Institute, German Aerospace Center (DLR), Oberpfaffenhofen, Germany. He has been working as Systems Engineer and Software Engineer for ground segments of radar satellite missions such as TerraSAR-X and TanDEM-X with focus on system design, integration and verification.

Mr. Grigorov was the recipient of an ESA Grant for the preparation of his master thesis about fine pointing methods for optical free-space communication in 2007, which was performed at DLR. He is a member of the International Council on Systems Engineering (INCOSE) since 2009 and a holder of the INCOSE Associate Systems Engineering Professional certificate. 